\title{
The partition function of the two-matrix model as an isomonodromic $\tau$ function
}

\author{
M. Bertola ${ }^{3,2, a)}$ and O. Marchal ${ }^{1,2, b)}$ \\ ${ }^{1}$ Institut de Physique Théorique, CEA, IPhT, F-91191 Gif-sur-Yvette, France and CNRS, \\ URA 2306, F-91191 Gif-sur-Yvette, France \\ ${ }^{2}$ Centre de Recherches Mathématiques, Université de Montréal, C. P. 6128, succ. centre \\ ville, Montréal, Québec H3C 3J7, Canada \\ ${ }^{3}$ Department of Mathematics and Statistics, Concordia University, 1455 de Maisonneuve \\ W., Montréal, Québec H3G 1M8, Canada
}

(Received 9 September 2008; accepted 1 December 2008; published online 15 January 2009)

We consider the Itzykson-Zuber-Eynard-Mehta two-matrix model and prove that the partition function is an isomonodromic $\tau$ function in a sense that generalizes that of Jimbo et al. [ "Monodromy preserving deformation of linear ordinary differential equations with rational coefficients," Physica D 2, 306 (1981)]. In order to achieve the generalization we need to define a notion of $\tau$ function for isomonodromic systems where the adregularity of the leading coefficient is not a necessary requirement. (C) 2009 American Institute of Physics. [DOI: 10.1063/1.3054865]

\section{INTRODUCTION}

Random matrix models have been studied for years and have generated important results in many fields of both theoretical physics and mathematics. ${ }^{1,16,18}$ The two-matrix model

$$
d \mu\left(M_{1}, M_{2}\right)=e^{-\operatorname{Tr}\left(V_{1}\left(M_{1}\right)+V_{2}\left(M_{2}\right)-M_{1} M_{2}\right)} d M_{1} d M_{2}
$$

was used to model two-dimensional quantum gravity ${ }^{14}$ and was investigated from a more mathematical point of view in Refs. 25, 17, 4, 5, 7, 12, and 8; the partition function of the model

$$
\mathcal{Z}_{N}\left(V_{1}, V_{2}\right)=\iint d \mu\left(M_{1}, M_{2}\right)
$$

has important properties in the large $N$ limit for the enumeration of discrete maps on surfaces ${ }^{15}$ of arbitrary genus and it is also known to be a $\tau$ function for the 2-Toda hierarchy. In the case of the Witten conjecture, proved by Kontsevich ${ }^{23}$ with the use of matrix integrals not too dissimilar from the above one, the enumerative properties of the $\tau$ function imply some nonlinear (hierarchy of) partial differential equations (PDEs) [the Korteweg-de Vries (KdV) hierarchy for the mentioned example]. On a similar level, one expects some hierarchy of PDEs for the case of the two-matrix model and possibly some Painlevé property (namely, the absence of movable essential singularities). The Painlevé property is characteristic of $\tau$ functions for isomonodromic families of ordinary differential equations (ODEs) that depend on parameters; hence, a way of establishing such property for the partition function $\mathcal{Z}_{N}$ is that of identifying it with an instance of isomonodromic $\tau$ function. ${ }^{20,21}$

This is precisely the purpose of this article; we capitalize on previous work that showed how to relate the matrix model to certain biorthogonal polynomials ${ }^{26,17,22,16}$ and how these appear in a natural fashion as the solution of certain isomonodromic family. ${ }^{9}$

\footnotetext{
${ }^{a)}$ Electronic mail: bertola@crm.umontreal.ca.

${ }^{\mathrm{b})}$ Electronic mail: olivier.marchal@ polytechnique.org.
} 
The paper extends to the case of the two-matrix model the work contained in Refs. 9, 12, and 10 ; it uses, however, a different approach closer to a recent one. ${ }^{6}$

In Refs. 9, 12, 10, and 19 the partition function of the one-matrix model (and certain shifted Töplitz determinants) were identified as isomonodromic $\tau$ functions by using spectral residue formulas in terms of the spectral curve of the differential equation. Such spectral curve has interesting properties inasmuch as-in the one-matrix case- the spectral invariants can be related to the expectation values of the matrix model. Recently the spectral curve of the two-matrix model $^{8}$ has been written explicitly in terms of expectation values of the two-matrix model and hence one could use their result and follow a similar path for the proof as the one followed in Ref. 12. Whichever one of the two approaches one chooses, a main obstacle is that the definition of isomonodromic $\tau$ function ${ }^{20,21}$ relies on a genericity assumption for the ODE which fails in the case at hand, thus requiring a generalization in the definition (see, however, Ref. 11 for a different generalization).

According to this logic, one of the purposes of this paper is to extend the notion of $\tau$ function introduced by Jimbo et al. ${ }^{20}$ to the two-matrix Itzykson-Zuber model. This task is accomplished in a rather general framework in Sec. III.

We then show that the partition function has a very precise relationship with the $\tau$ function so introduced, allowing us to (essentially) identify it as an isomonodromic $\tau$ function (Theorem 3.4). The proof is organized as follows. In Sec. II we recall a Riemann-Hilbert formulation of the problem due to Ref. 24. In Sec. II B we transform it into a Riemann-Hilbert problem (RHP) with constant jumps, thus guaranteeing the existence of an ODE. As the asymptotic at infinity does not enter into the class of the Jimbo-Miwa theorem for isomonodromic $\tau$ function, we generalize their result to our setting in Sec. III. Using Schlesinger transformations, namely, the shift in the size of the matrix model from $N$ to $N+1$, we eventually show that the partition function equals the new defined isomonodromic $\tau$ function in Sec. III B.

\section{A RIEMANN-HILBERT FORMULATION OF THE TWO-MATRIX MODEL}

According to a seminal work ${ }^{26,17}$ and following the notations and definitions introduced in Refs. 4 and 5, we consider paired sequences of monic polynomials $\left\{\pi_{m}(x), \sigma_{m}(y)\right\}_{m=0, \ldots, \infty}(m$ $=\operatorname{deg} \pi_{m}=\operatorname{deg} \sigma_{m}$ ) that are biorthogonal in the sense that

$$
\iint_{\varkappa} d x d y \pi_{m}(x) \sigma_{n}(y) e^{-V_{1}(x)-V_{2}(y)+x y}=h_{m} \delta_{m n}, \quad h_{m} \neq 0 .
$$

The functions $V_{1}(x)$ and $V_{2}(y)$ appearing here are referred to as potentials, terminology drawn from random matrix theory, in which such quantities play a fundamental role.

Henceforth, the second potential $V_{2}(y)$ will be chosen as a polynomial of degree $d_{2}+1$,

$$
V_{2}(y)=\sum_{J=1}^{d_{2}+1} \frac{v_{J}}{J} y^{J}, \quad v_{d_{2}+1} \neq 0 .
$$

For the purposes of most of the considerations to follow, the first potential $V_{1}(x)$ may have very general analyticity properties as long as the manipulations make sense, but for definiteness and clarity, we choose it to be polynomial as well:

$$
V_{1}(x)=\sum_{K=1}^{d_{1}+1} \frac{u_{K}}{K} x^{K}, \quad u_{d_{1}+1} \neq 0 .
$$

The symbol $\iint_{\varkappa}$ stands for any linear combination of integrals of the form 


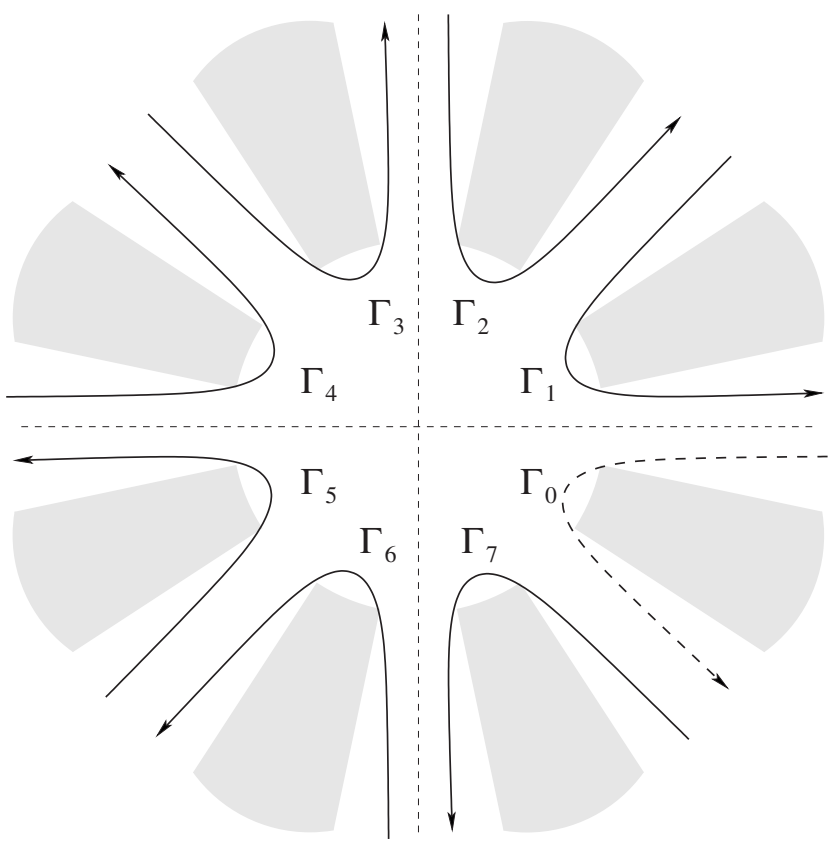

FIG. 1. Wedge and antiwedge contours for $V_{2}(y)$ of degree $d_{2}+1$ (=8 in this example).

$$
\iint_{\varkappa} d x d y:=\sum_{j} \sum_{k} x_{j k} \int_{\Gamma_{j}} d x \int_{\hat{\Gamma}_{k}} d y, \quad x_{i j} \in \mathrm{C},
$$

where the contours $\left\{\hat{\Gamma}_{k}\right\}_{k=1, \ldots, d_{2}}$ will be chosen as follows. In the $y$ plane, define $d_{2}+1$ "wedge sectors" $\left\{\hat{S}_{k}\right\}_{k=0, \ldots, d_{2}}$ such that $\hat{S}_{k}$ is bounded by the pairs of rays $r_{k}:=\left\{y \mid \arg y=\theta+2 k \pi /\left(d_{2}+1\right)\right\}$ and $r_{k-1}:=\left\{y \mid \arg y=\theta+2(k-1) \pi /\left(d_{2}+1\right)\right\}$, where $\theta:=\arg v_{d_{2}+1}$. Then $\hat{\Gamma}_{k}$ is any smooth oriented contour within the sector $\hat{S}_{k}$ starting from $\infty$ asymptotic to the ray $r_{k}$ [or any ray within the sector that is at an angle $<\pi / 2\left(2 d_{2}+1\right)$ to it, which is equivalent for purposes of integration] and returning to $\infty$ asymptotically along $r_{k-1}$ [or at an angle $<\pi / 2\left(2 d_{2}+1\right)$ to it]. These will be referred to as the "wedge contours." We also define a set of smooth oriented contours $\left\{\check{\Gamma}_{k}\right\}_{k=1, \ldots, d_{2}}$ that have intersection matrix $\check{\Gamma}_{j} \cap \hat{\Gamma}_{k}=\delta_{j k}$ with the $\hat{\Gamma}_{k}$ such that $\check{\Gamma}_{k}$ starts from $\infty$ in sector $\hat{S}_{0}$, asymptotic to the ray $\check{r}_{0}:=\left\{y \mid \arg (y)=\theta-\pi /\left(d_{2}+1\right)\right.$ and returns to $\infty$ in sector $\hat{S}_{k}$ asymptotically along the ray $\check{r}_{k}:=\left\{y \mid \arg (y)=\theta+2(k-1 / 2) /\left(d_{2}+1\right)\right.$. These will be called the "antiwedge" contours. (See Fig. 1.) The choice of these contours is determined by the requirement that all moment integrals of the form

$$
\int_{\hat{\Gamma}_{k}} y^{j} e^{-V_{2}(y)+x y} d y, \quad \int_{\check{\Gamma}_{k}} y^{k} e^{V_{2}(y)-x y} d y, \quad k=1, \ldots, d_{2}, \quad j \in \mathbb{N},
$$

be uniformly convergent in $x \in \mathrm{C}$. In the case when the other potential $V_{1}(x)$ is also a polynomial, of degree $d_{1}+1$, the contours $\left\{\Gamma_{k}\right\}_{k=1, \ldots, d_{1}}$ in the $x$ plane may be defined similarly.

The partition function is defined here to be the multiple integral

$$
\mathcal{Z}_{N}:=\frac{1}{N !} \iint_{\chi^{N}} \prod_{j=1}^{N} d x_{j} d y_{j} \Delta(X) \Delta(Y) \prod_{j=1}^{N} e^{-V_{1}\left(x_{j}\right)-V_{2}\left(y_{j}\right)+x_{j} y_{j}},
$$

where $\Delta(X)$ and $\Delta(Y)$ denote the usual Vandermonde determinants and the factor $1 / N$ ! is chosen for convenience. 
Such multiple integral can also be represented as the following determinant:

$$
\mathcal{Z}_{N}=\operatorname{det}\left[\mu_{i j}\right]_{0 \leq i, j \leq N-1}, \quad \mu_{i j}:=\int_{\varkappa} x^{i} y^{j} e^{-V_{1}(x)-V_{2}(y)+x y} d x d y .
$$

The denomination of the partition function comes from the fact ${ }^{26,17,9}$ that when $\varkappa$ coincides with $\mathbb{R} \times \mathbb{R}$ then $\mathcal{Z}_{N}$ coincides (up to a normalization for the volume of the unitary group) with the following matrix integral:

$$
\iint d M_{1} d M_{2} e^{-\operatorname{tr}\left(V_{1}\left(M_{1}\right)+V_{2}\left(M_{2}\right)-M_{1} M_{2}\right)},
$$

extended over the space of Hermitian matrices $M_{1}$ and $M_{2}$ of size $N \times N$, namely, the normalization factor for the measure $d \mu\left(M_{1}, M_{2}\right)$ introduced in (1.1).

\section{A. Riemann-Hilbert characterization for the orthogonal polynomials}

A Riemann-Hilbert characterization of the biorthogonal polynomials is a crucial step toward implementing a steepest-descent analysis. In our context it is also crucial in order to tie the random matrix side to the theory of isomonodromic deformations.

We first recall the approach given by Kuijlaars and McLaughin (referred to as KM in the rest of the article) in Ref. 24 suitably extended and adapted (in a rather trivial way) to the setting and notation of the present work. We quote-paraphrasing and with a minor generalization-their theorem without proof.

Theorem 2.1: $\left(\mathrm{KM}\right.$ asymptotic $\left.{ }^{24}\right)$ The monic biorthogonal polynomial $\pi_{n}(x)$ is the $(1,1)$ entry of the solution $\Gamma(x)$ (if it exists) of the following Riemann-Hilbert problem for $\Gamma(x)$.

(1) The matrix $\Gamma(x)$ is piecewise analytic in $\mathrm{C \backslash} \sqcup \Gamma_{j}$.

(2) The (nontangential) boundary values of $\Gamma(x)$ satisfy the relations

$$
\begin{gathered}
\Gamma(x)_{+}=\Gamma(x)_{-}\left[\begin{array}{cccc}
1 & w_{j, 1} & \cdots & w_{j, d_{2}} \\
& 1 & 0 & 0 \\
& \ddots & \\
& & & 1
\end{array}\right], \quad x \in \Gamma_{j}, \\
w_{j, \nu}=w_{j, \nu}(x):=e^{-V_{1}(x)} \sum_{k=1}^{d_{2}} x_{j k} \int_{\hat{\Gamma}_{k}} y^{\nu-1} e^{-V_{2}(y)+x y} d y .
\end{gathered}
$$

(3) As $x \rightarrow \infty$, we have the following asymptotic expansion:

$$
\Gamma(x) \sim\left(I_{d}+\frac{Y_{N, 1}}{x}+\mathcal{O}\left(\frac{1}{x^{2}}\right)\right)\left(\begin{array}{ccc}
x^{N} & 0 & 0 \\
0 & x^{-m_{N}-1} I d_{r_{N}} & 0 \\
0 & 0 & x^{-m_{N} I d_{d_{2}-r_{N}}}
\end{array}\right),
$$

where we have defined the integers $m_{N}$ and $r_{N}$ as follows:

$$
N=m_{N} d_{2}+r_{N}, \quad m_{N}, r_{N} \in \mathbb{N}, \quad 0 \leq r_{n} \leq d_{2}-1 .
$$

It follows from Ref. 24 that the solution $\Gamma_{N}(x)$ has the following form: 


$$
\begin{aligned}
\Gamma_{N}(x):=\Gamma(x) & :=\left[\begin{array}{cccc}
\pi_{N}(x) & \mathcal{C}_{0}\left(\pi_{N}\right) & \cdots & \mathcal{C}_{d_{2}-1}\left(\pi_{N}\right) \\
p_{N-1}(x) & \mathcal{C}_{0}\left(p_{N-1}\right) & \cdots & \mathcal{C}_{d_{2}-1}\left(p_{N-1}\right) \\
\vdots & & & \vdots \\
p_{N-d_{2}}(x) & \mathcal{C}_{0}\left(p_{N-d_{2}}\right) & \cdots & \mathcal{C}_{d_{2}-1}\left(p_{N-d_{2}}\right)
\end{array}\right], \\
\mathcal{C}_{i}(f(z)) & :=\frac{1}{2 \pi i} \iint_{\varkappa} \frac{f(x)}{x-z} y^{i} e^{-V_{1}(x)-V_{2}(y)+x y} d y d x,
\end{aligned}
$$

where the polynomials denoted above by $p_{N-1}, \ldots, p_{N-d_{2}}$ are some polynomials of degree not exceeding $N-1$, whose detailed properties are largely irrelevant for our discussion; we refer to Ref. 24 for these details.

By a left multiplication of this solution by a suitable constant matrix (i.e., some suitable linear combination of the last rows), we can see that the matrix

$$
\hat{\Gamma}_{N}:=\left[\begin{array}{cccc}
\pi_{n} & \mathcal{C}_{0}\left(\pi_{n}\right) & \cdots & \mathcal{C}_{d_{2}-1}\left(\pi_{n}\right) \\
\pi_{n-1} & \mathcal{C}_{0}\left(\pi_{n-1}\right) & \cdots & \mathcal{C}_{d_{2}-1}\left(\pi_{n-1}\right) \\
\vdots & & & \vdots \\
\pi_{n-d_{2}} & \mathcal{C}_{0}\left(\pi_{n-d_{2}}\right) & \cdots & \mathcal{C}_{d_{2}-1}\left(\pi_{n-d_{2}}\right)
\end{array}\right]
$$

and $\Gamma_{N}$ are related as

$$
\hat{\Gamma}_{N}(x)=U_{N} \Gamma_{N}(x),
$$

where $U_{N}$ is a constant matrix (depending on $N$ and on the coefficients of the polynomials but not on $x$ ). As an immediate consequence, $\hat{\Gamma}_{N}$ solves the same RHP as $\Gamma$ with the exception of the normalization at infinity (2.11). The matrix $U_{N}$ will not play a major role in the following proof, but we mention it for completeness.

The RHP featured in Theorem 2.1 is not immediately suitable to make the connection to the theory of isomonodromic deformations as described in Refs. 20 and 21; we recall that this is the theory that describes the deformations of an ODE in the complex plane which leave the Stokes matrices (i.e., the so-called extended monodromy data) invariant. The solution $\Gamma_{N}\left(\right.$ or $\hat{\Gamma}_{N}$ ) does not solve any ODE as formulated because the jumps on the contours are nonconstant. If, however, we can relate $\Gamma_{N}$ with some other RHP with constant jumps, then its solution can be immediately shown to satisfy a polynomial ODE, which allows us to use the machinery of Refs. 20 and 21. This is the purpose of Sec. III A.

\section{B. A RHP with constant jumps}

In Ref. 9 the biorthogonal polynomials were characterized in terms of an ODE or-which is the same- of a RHP with constant jumps. In order to connect the two formulations, we will use some results contained in Ref. 13, and we start by defining some auxiliary quantities: for $1 \leq k$ $\leq d_{2}$, define the $d_{2}$ sequences of functions $\left\{\psi_{m}^{(k)}(x)\right\}_{m \in \mathbb{N}}$ as follows:

$$
\psi_{m}^{(k)}(x):=\frac{1}{2 \pi i} \int_{\check{\Gamma}_{k}} d s \int_{\varkappa} d z d w \frac{\pi_{m}(z) e^{-V_{1}(z)}}{x-z} \frac{V_{2}^{\prime}(s)-V_{2}^{\prime}(w)}{s-w} e^{-V_{2}(w)+V_{2}(s)+z w-x s}, \quad 1 \leq k \leq d_{2},
$$

and let

$$
\psi_{m}^{(0)}(x):=\pi_{m}(x) e^{-V_{1}(x)}
$$



$\widehat{\Psi_{N}}(x)$,

In terms of these define, for $N \geq d_{2}$, the sequence of $\left(d_{2}+1\right) \times\left(d_{2}+1\right)$ matrix valued functions

$$
\hat{\Psi}(x):=\left[\begin{array}{ccc}
\psi_{N}^{(0)}(x) & \cdots & \psi_{N}^{\left(d_{2}\right)}(x) \\
\vdots & & \vdots \\
\psi_{N-d_{2}}^{(0)}(x) & \cdots & \psi_{N-d_{2}}^{\left(d_{2}\right)}(x)
\end{array}\right] .
$$

For convenience (the notation with a hat describes only that we have removed the normalization matrix $U_{N}$ ), we define also

$$
\underset{N}{\Psi}:=U_{N}^{-1} \hat{\Psi} .
$$

The relationship with the matrices $\Gamma_{N}$ and $\hat{\Gamma}_{N}$ introduced in the previous section is detailed in the following crucial theorem.

Theorem 2.2: (Factorization theorem) The following identities hold:

$$
\begin{gathered}
\underset{N}{\Psi}(x)=\hat{\Gamma}_{N}(x) \Psi_{0}(x), \quad \underset{N}{\Psi}(x)=\Gamma_{N}(x) \Psi_{0}(x), \\
\Psi_{0}(x):=V(x) W(x),
\end{gathered}
$$

where

$$
V:=\left(\begin{array}{cc}
e^{-V_{1}(x)} & 0 \\
0 & V_{0},
\end{array}\right), \quad W(x):=\left(\begin{array}{cc}
1 & 0 \\
0 & W_{0}(x)
\end{array}\right)
$$

and $V_{0}$ and $W_{0}(x)$ are the $d_{2} \times d_{2}$ matrices with elements

$$
\begin{aligned}
\left(V_{0}\right)_{j k} & =\left[\begin{array}{cccc}
v_{2} & v_{3} & \cdots & \\
v_{3} & & & v_{d_{2}+1} \\
& & . & \\
v_{d_{2}} & v_{d_{2}+1} \\
v_{d_{2}+1} &
\end{array}\right] \\
& = \begin{cases}v_{j+k} & \text { if } j+k \leq d_{2}+1 \\
0 & \text { if } j+k>d_{2}+1,\end{cases} \\
\left(W_{0}(x)\right)_{j k} & =\int_{\check{\Gamma}_{k}}^{y^{j-1} e^{V_{2}(y)-x y} d y, \quad 1 \leq j, \quad k \leq d_{2} .}
\end{aligned}
$$

Proof: The proof is contained in Appendix A (based on Refs. 13 and 3) and is a direct verification based on matrix multiplication, noticing that the matrix $V_{0}$ is nothing but the matrix representation of $\left(V_{2}^{\prime}(y)-V_{2}^{\prime}(s)\right) /(y-s)$ as a quadratic form in the bases $1, y, y^{2}, \ldots, y^{d_{2}-1}$ and $1, s, s^{2}, \ldots, s^{d_{2}-1}$.

The point of the matter now is that this new matrix $\Psi_{N}$ (or $\widehat{\Psi_{N}}$ ) solves a Riemann-Hilbert problem with constant jumps; this is due to the right multiplication of the factor $\Psi_{0}:=V(x) W(x)$. To this end, we state the following theorem.

Theorem 2.3: The matrix $\Psi_{N}$ is the unique solution of the following RHP.

(1) Constant jumps: 


$$
\underset{N}{\Psi_{+}}(x)=\underset{N}{\Psi_{-}}(x) \mathbf{H}^{(j)}, \quad x \in \Gamma_{j},
$$

where

$$
\begin{gathered}
\mathbf{H}^{(j)}:=\mathbf{I}-2 \pi i e_{0} \kappa^{T}, \\
\hat{\mathbf{H}}^{(j)}=\left(\mathbf{H}^{(j)}\right)^{-1}=\mathbf{I}+2 \pi i e_{0} \kappa^{T}, \\
e_{0}:=\left(\begin{array}{c}
1 \\
0 \\
\vdots \\
0
\end{array}\right), \quad \kappa:=\left(\begin{array}{c}
0 \\
\varkappa_{j 1} \\
\vdots \\
\varkappa_{j d_{2}}
\end{array}\right) .
\end{gathered}
$$

(2) Asymptotic behavior at infinity:

$$
\Psi(x) \sim \Gamma_{N}\left(\begin{array}{ccc}
x^{N} e^{-V_{1}(x)} & 0 & 0 \\
0 & x^{-m_{N}-1} I d_{r_{N}} & 0 \\
0 & 0 & x^{-m_{N} I d_{d_{2}-r_{N}}}
\end{array}\right) \Psi_{0}(x),
$$

where

$$
\Gamma_{N}=I d+\frac{Y_{N, 1}}{x}+\cdots
$$

and where $\Psi_{0}(x):=V(x) W(x)$ will be referred to as the bare solution. Its asymptotic at infinity can be computed by steepest descent, but since it is $N$ independent, for the sake of brevity, we do not report on it (details are contained in Refs. 5 and 13).

(3) $\Psi_{N}^{\prime}(x) \Psi_{N}^{-1}=D_{N}(x)$ where $D_{N}(x)$ is a polynomial in $x$ of degree $d_{1}$.

(4) $\partial_{u_{K}} \Psi_{N}(x) \Psi_{N}^{-1}=U_{K, N}(x)$ is polynomial in $x$ of degree $K$.

(5) $\partial_{v_{J}} \Psi_{N}(x) \Psi_{N}^{-1}=V_{J, N}(x)$ is polynomial in $x$.

(6) $\operatorname{det}\left(\Psi_{N+1} \Psi_{N}^{-1}\right) \equiv C$ with $C$ a constant independent of $x$.

(Sketch of) Proof: In order to prove point (1), we have to directly consider the definition of $\widehat{\Psi_{N}}$ (noticing that $\Psi_{N}$ has the same jumps since they differ only by a left multiplier). Using Sokhotski-Plemelji formulas and the results of Appendix B, one can quickly obtain the proof. Point (2) is a consequence of the factorization Theorem 2.2 and the asymptotic representation of $\Gamma_{N}$ given in Theorem 2.1.

The uniqueness of the solution is proved by showing that det $\Psi_{N}$ is constant in $x$; this follows from the fact that the determinant has no jumps due to the unimodularity of the jump matrices, and the fact that $\operatorname{det} \Psi_{0}$ is constant and at infinity $\operatorname{det} \Psi_{N}$ tends to a constant. Thus, by Liouville's theorem det $\Psi_{N}$ must be constant. At this point, the argument goes that any other solution $\widetilde{\Psi}_{N}$ must be invertible as well; the ratio $\Psi_{N} \widetilde{\Psi}_{N}{ }^{-1}$ has no jump, thus being entire, and tends to the identity at infinity. Once more, Liouville's theorem assures that then the two matrices are identically equal. The points (3)-(6) in the above theorem can be found in Refs. 8 and 5, and the proofs will not be repeated here in detail. For example, point (3) can be proven by noticing that since $\Psi_{N}$ has constant jumps, the expression $\Psi_{N}^{\prime} \Psi_{N}^{-1}$ has no jumps and is hence entire. The fact that it is in fact a polynomial of degree $d_{1}$ follows from the asymptotic behavior at infinity. In a similar fashion, one can prove the other two points.

The main upshot of points (3)-(5) is that $\Psi_{N}$ is the solution of an isomonodromic deformation system: namely, we may think of the equations as deformation equations which leave the (generalized) monodromy of the ODE in (3) invariant, as defined in Refs. 20 and 21. We can thus apply the ideas of the said papers to our situation after the suitable generalizations. 
In the next section, we shall define a proper notion of isomonodromic $\tau$ function: it should be pointed out that the definition of Refs. 20 and 21 cannot be applied as such because-as shown in Ref. 5- the ODE that the matrix $\Psi_{N}\left(\right.$ or $\left.\widehat{\Psi_{N}}\right)$ solves has a highly degenerate leading coefficient at the singularity at infinity.

In the list, the crucial ingredients are the differential equations (in $x$ or relatively to the parameters $u_{K}$ and $\left.v_{J}\right)$. First, the fact that $D_{N}(x)$ is a polynomial comes from explicit computation (see Ref. 8, for example). The result concerning the determinant of $R_{N}(x)$ can also be found in Ref. 8 where one has $\operatorname{det}\left(\Psi_{N+1} \Psi_{N}^{-1}\right)=\operatorname{det}\left(a_{N}(x)\right)=C$ ste. The properties concerning the differential equations relatively to parameters can be found in Ref. 8 too. Under all these assumptions, we will show that the proof of Jimbo et al. can be adapted and that we can define a suitable $\tau$ function in the same way Jimbo et al. did.

\section{DEFINITION OF THE $\tau$ FUNCTION}

In this section, we will place ourselves in a more general context than the one described above; we will show that, under a few assumptions, one can define a good notion of $\tau$ function.

More generally, we will denote with $t_{a}$ the isomonodromic parameters or "isomonodromic times" (in our case they are the $u_{K}$ and the $v_{J}$ ) and a subscript $a$ or $b$ is understood as a derivation relative to $t_{a}$ or $t_{b}$. For a function $f$ of the isomonodromic times, we will denote by the usual symbol its differential,

$$
d f=\sum_{a} \partial_{t_{d}} f d t_{a}=\sum_{a} f_{a} d t_{a}
$$

Our setup falls in the following framework that it is useful to ascertain from the specifics of the case at hand. Suppose we are given a matrix whose behavior at infinity is

$$
\Psi(x) \sim Y(x) \Xi(x), \quad Y(x):=\left(1+\frac{Y_{1}}{x}+\frac{Y_{2}}{x^{2}}+\cdots\right) x^{S},
$$

where $\Xi(x)=\Xi(x ; \mathbf{t})$ is some explicit expression (the "bare" isomonodromic solution) and $S$ is a matrix independent of the isomonodromic times. Note that this asymptotic falls exactly in the one we get for $\Psi_{N}(x)$. This implies that if we define the one-form-valued matrix $\mathcal{H}(x ; \mathbf{t})$ by

$$
\mathcal{H}(x ; \mathbf{t})=d \Xi(x ; \mathbf{t}) \Xi(x ; \mathbf{t})^{-1}
$$

then $\mathcal{H}(x)=\Sigma \mathcal{H}_{a} d t_{a}$ (we suppress explicit mention of the $\mathbf{t}$ dependence henceforth) is some solution of the zero-curvature equations:

$$
\partial_{a} \mathcal{H}_{b}-\partial_{b} \mathcal{H}_{a}=\left[\mathcal{H}_{a}, \mathcal{H}_{b}\right]
$$

This result is a direct computation using $\mathcal{H}_{a}=\left(\partial_{a} \Xi\right) \Xi^{-1}$ and $\partial_{b}\left(\Xi^{-1}\right)=-\Xi^{-1}\left(\partial_{b} \Xi\right) \Xi^{-1}$. We will assume (which is the case in our setting) that all $\mathcal{H}_{a}$ are polynomials in $x$. We will also use that the dressed deformations $\Omega_{a}$ given by $\Psi_{a}=\Omega_{a} \Psi$ are polynomials, which is the case in our setup, thanks to properties (4) and (5) of Theorem 2.3. Moreover, according to the asymptotic, they are given by

$$
\Omega_{a}=\left(Y \mathcal{H}_{a} Y^{-1}\right)_{\mathrm{pol}} .
$$

In this very general (and generic) setting, we can formulate the definition of a " $\tau$ function" as follows.

Definition 3.1: The $\tau$ differential is the one-form defined by

$$
\omega:=\sum \omega_{a} d t^{a}:=\sum_{a} \operatorname{res} \operatorname{tr}\left(Y^{-1} Y^{\prime} \mathcal{H}_{a}\right) d t^{a} .
$$


The main point of the matter is that-without any further details-we can now prove that the $\tau$ differential is in fact closed and hence locally defines a function.

Theorem 3.1: The $\tau$ differential is a closed differential and locally defines a $\tau$ function as

$$
d \log \tau=\omega .
$$

Proof: We need to prove the closure of the differential. We first recall the main relations between the bare and dressed deformations,

$$
\partial_{a} Y=\Omega_{a} Y-Y \mathcal{H}_{a}, \quad Y \mathcal{H}_{a} Y^{-1}=\Omega_{a}-\mathcal{R}_{a}, \quad \mathcal{R}_{a}:=\partial_{a} Y Y^{-1} .
$$

We note that-by construction- $\Omega_{a}=\left(Y \mathcal{H}_{a} Y^{-1}\right)_{\text {pol }}$ is a polynomial while $\mathcal{R}_{a}=\mathcal{O}\left(x^{-1}\right)$ irrespective of the form of $S$. We compute the cross derivatives directly,

$$
\begin{aligned}
\partial_{a} \omega_{b} & =\operatorname{res} \operatorname{Tr}\left(-Y^{-1}\left(\Omega_{a} Y-Y \mathcal{H}_{a}\right) Y^{-1} Y^{\prime} \mathcal{H}_{b}+Y^{-1}\left(\Omega_{a} Y-Y \mathcal{H}_{a}\right)^{\prime} \mathcal{H}_{b}+Y^{-1} Y^{\prime} \partial_{a} \mathcal{H}_{b}\right) \\
& =\operatorname{res} \operatorname{Tr}\left(\mathcal{H}_{a} Y^{-1} Y^{\prime} \mathcal{H}_{b}+Y^{-1} \Omega_{a}^{\prime} Y \mathcal{H}_{b}-Y^{-1} Y^{\prime} \mathcal{H}_{a} \mathcal{H}_{b}-\mathcal{H}_{a}^{\prime} \mathcal{H}_{b}+Y^{-1} Y^{\prime} \partial_{a} H_{b}\right) \\
& =\operatorname{polynomial}
\end{aligned}
$$

where, in the last step, we have used that $Y \mathcal{H}_{b} Y^{-1}=\Omega_{b}-\mathcal{R}_{b}$ and that the contribution coming from $\Omega_{b}$ vanishes since it is a polynomial. Rewriting the same with $a \leftrightarrow b$ and subtracting, we obtain

$$
\begin{aligned}
\partial_{a} \omega_{b}-\partial_{b} \omega_{a} & =\operatorname{res} \operatorname{Tr}\left(2 Y^{-1} Y^{\prime}\left[\mathcal{H}_{b}, \mathcal{H}_{a}\right]-\Omega_{a}^{\prime} \mathcal{R}_{b}+\Omega_{b}^{\prime} \mathcal{R}_{a}+Y^{-1} Y^{\prime}\left(\partial_{a} \mathcal{H}_{b}-\partial_{b} \mathcal{H}_{a}\right)\right) \\
& \left.=\operatorname{res} \operatorname{Tr}\left(Y^{-1} Y^{\prime}\left[\mathcal{H}_{b}, \mathcal{H}_{a}\right]-\Omega_{a}^{\prime} \mathcal{R}_{b}+\Omega_{b}^{\prime} \mathcal{R}_{a}+Y^{-1} Y^{\prime}(\overbrace{\partial_{a} \mathcal{H}_{b}-\partial_{b} \mathcal{H}_{a}+\left[\mathcal{H}_{b}, \mathcal{H}_{a}\right.}]\right)\right) \\
& =\operatorname{res} \operatorname{Tr}\left(Y^{-1} Y^{\prime}\left[\mathcal{H}_{b}, \mathcal{H}_{a}\right]-\Omega_{a}^{\prime} \mathcal{R}_{b}+\Omega_{b}^{\prime} \mathcal{R}_{a}\right) .
\end{aligned}
$$

Note that, up to this point, we only used the zero-curvature equations for the connection $\nabla$ $=\Sigma\left(\partial_{a}-\mathcal{H}_{a}\right) d t^{a}$ and the fact that $\mathcal{H}_{a}$ are polynomials in $x$. We thus need to prove that the last quantity in (3.10) vanishes: this follows from the following computation, which uses once more the fact that $\mathcal{H}_{a}$ and $\Omega_{a}$ are all polynomials. Indeed, we have res $\operatorname{Tr}\left(\mathcal{H}_{a}^{\prime} \mathcal{H}_{b}\right)=0$ and hence [using (3.8)]

$$
\begin{aligned}
0 & =\operatorname{res} \operatorname{tr}\left(\mathcal{H}_{a}^{\prime} \mathcal{H}_{b}\right)=\operatorname{res} \operatorname{Tr}\left(\left(Y \mathcal{H}_{a} Y^{-1}\right)^{\prime} Y \mathcal{H}_{b} Y^{-1}\right)-\operatorname{res} \operatorname{Tr}\left(Y^{\prime} \mathcal{H}_{a} \mathcal{H}_{b} Y^{-1}\right)+\operatorname{res} \operatorname{Tr}\left(\mathcal{H}_{a} Y^{-1} Y^{\prime} \mathcal{H}_{b}\right) \\
& =\operatorname{res} \operatorname{Tr}\left(\left(\Omega_{a}-\mathcal{R}_{a}\right)^{\prime}\left(\Omega_{b}-\mathcal{R}_{b}\right)\right)+\operatorname{res} \operatorname{Tr}\left(Y^{-1} Y^{\prime}\left[\mathcal{H}_{b}, \mathcal{H}_{a}\right]\right) \\
& =\mathcal{O}\left(x^{-2}\right)
\end{aligned}
$$

Using integration by parts (and cyclicity of the trace) on the first term here above, we obtain precisely the last quantity in (3.10). The theorem is proved.

\section{A. Application to our problem}

We now apply the general definition above to our setting, with the identifications $\Psi=\Psi_{N}$, $Y=\Gamma_{N}$ (as a formal power series at $\infty$ ), and $\Xi=\Psi_{0}$. We will write $Y_{N}$ instead of $\Gamma_{N}$ in the expressions below to emphasize that we consider its asymptotic expansion at $\infty$. In order to apply the generalized Definition 3.1 to our setting, we identify

- $\Gamma_{N}$ of Theorem 2.3 with $Y$ of Eq. (3.2), 
- the times $t_{a}$ used in Sec. III with the coefficients $v_{j}$ and $u_{K}$ of the two potentials [(2.3) and (2.2)], and

- the "bare isomonodromic solution" $\Xi(x ; \mathbf{t})$ of Sec. III with the bare solution $\Psi_{0}(x ; \vec{u}, \vec{v})$ appearing in Theorem 2.3.

This reduces the definition of the $\tau$ function to the one below.

Definition 3.2: The $\tau$ function is defined by the following PDE:

$$
d\left(\log \tau_{N}\right)=\underset{x \rightarrow \infty}{\operatorname{Res}} \operatorname{Tr}\left(Y_{N}^{-1} Y_{N}^{\prime} \mathrm{d}\left(\Psi_{0}\right) \Psi_{0}^{-1}\right),
$$

where $Y_{N}$ is the formal asymptotic expansion of $\Gamma_{N}$ at infinity,

$$
Y_{N}=\tilde{Y}_{N}\left(\begin{array}{ccc}
x^{N} & 0 & 0 \\
0 & x^{-m_{N}-1} I d_{r_{N}} & 0 \\
0 & 0 & x^{-m_{N} I d_{d_{2}-r_{N}}}
\end{array}\right) .
$$

Remark 3.1: The matrix $S$ of the previous section [Eq. (3.2)] in our case becomes

$$
S=S_{N}=\left(\begin{array}{ccc}
N & 0 & 0 \\
0 & \left(-m_{N}-1\right) I d_{r_{N}} & 0 \\
0 & 0 & -m_{N} I d_{d_{2}-r_{N}}
\end{array}\right)
$$

as follows by inspection of Eq. (3.13).

The partial derivatives of $\ln \tau_{N}$ with respect to the times $u_{K}$ and $v_{J}$ split into two sets which have different forms:

$$
\begin{gathered}
\partial_{u_{K}} \log \tau_{N}=-\operatorname{Res}_{x \rightarrow \infty} \operatorname{Tr}\left(Y_{N}^{-1} Y_{N}^{\prime} \frac{x^{K}}{K} \mathbf{E}_{\mathbf{1 1}}\right), \\
\partial_{v_{J}} \log \tau_{N}=\operatorname{Res}_{x \rightarrow \infty} \operatorname{Tr}\left(Y_{N}^{-1} Y_{N}^{\prime} \partial_{v_{J}}\left(\Psi_{0}\right) \Psi_{0}^{-1}\right) .
\end{gathered}
$$

These two equations are the specializations of Definition 3.1 using that $\Xi(x ; \mathbf{t})=\Psi_{0}(x ; \vec{u}, \vec{v})$ and thus $\mathcal{H}(x)=\mathrm{d} \Xi \Xi^{-1}$ take on two forms, depending on which of the times we are differentiating with. The block form of the derivatives $\partial_{u_{K}} \Psi_{0} \Psi_{0}^{-1}$ or $\partial_{v_{J}} \Psi_{0} \Psi_{0}^{-1}$ follows simply by the block form of $\Psi_{0}$ (in Theorem 2.2), noticing that the dependence on the $u_{K}$ is only in the $(1,1)$ entry $\left[\left(\Psi_{0}\right)_{11}\right.$ $\left.=e^{-V_{1}(x)}\right]$, while the dependence on the $v_{J}$ is only in the $d_{2} \times d_{2}$ lower-right block.

One can notice that the situation we are looking at is a generalization of what happens in the one-matrix case. In the one-matrix model, the matrix $S$ is zero and therefore $Y_{N}$ are (formal) Laurent series. The matrix $\Psi_{0}$ matrix is absent in that case since there is only one potential, and thus one recovers the usual definition of isomonodromic $\tau$ function (see Ref. 12). Note also that in the derivation with respect to $v_{J}$, we have obtained the second equality using the block diagonal structure of $\Psi_{0}$ (first row/column does not play a role). It is remarkable that the two systems are completely decoupled, i.e., that in the first one the matrix $\Psi_{0}$ (containing all the dependence in $V_{2}$ ) disappears and that in the second one the matrix $A_{0}$ (containing the potential $V_{1}$ ) also disappears. Now that we have defined a proper generalization of isomonodromic $\tau$ functions corresponding to our setting; we still need to make the link with the partition function.

\section{B. Discrete Schlesinger transformation: $\tau$ function quotient}

In this section, we investigate the relationship between the $\tau$ function of Definition 3.2 and the partition function $\mathcal{Z}_{N}$ of the matrix model. We anticipate that the two objects turn out to be the same (up to a nonzero factor that will be explicitly computed, Theorem 3.4): the proof relies on two steps, the first of which we prepare in this section. These are 
- proving that they satisfy the same recurrence relation in $N$ and

- identifying the initial conditions for the recurrence relation.

We start by investigating the relationship between $\tau_{N}$ and $\tau_{N+1}$; this analysis is essentially identical to the theory developed in Ref. 21 and used in and Ref. 6, but we report it here for the convenience of the reader.

From the fact that the $\Psi_{N}$ has constant jumps, we deduce that $\Psi_{N+1} \Psi_{N}^{-1}$ is an entire function. Moreover, asymptotically it looks like

$$
\begin{gathered}
\Psi_{N+1} \Psi_{N}^{-1}=\tilde{Y}_{N+1}\left(\begin{array}{ccc}
x^{N+1} e^{-V_{1}(x)} & 0 & 0 \\
0 & x^{-m_{N+1}-1} I d_{r_{N+1}} & 0 \\
0 & 0 & x^{-m_{N+1} I} d_{d_{2}-r_{N+1}}
\end{array}\right) \Psi_{0}(x), \\
\Psi_{0}(x)^{-1}\left(\begin{array}{ccc}
x^{-N} e^{V_{1}(x)} & 0 & 0 \\
0 & x^{m_{N}+1} I d_{r_{N}} & 0 \\
0 & 0 & x^{m_{N} I d_{d_{2}-r_{N}}}
\end{array}\right) \tilde{Y}_{N}, \\
\Psi_{N+1} \Psi_{N}^{-1}=\tilde{Y}_{N+1}\left(\begin{array}{cccc}
x & 0 & 0 & 0 \\
0 & I d_{r_{N}-1} & 0 & 0 \\
0 & 0 & x^{-1} & 0 \\
0 & 0 & 0 & I d_{d_{2}-1-r_{N}}
\end{array}\right) \tilde{Y}_{N} .
\end{gathered}
$$

Thus, remembering that $\widetilde{Y}_{N}$ is a series $x^{-1}$, Liouville's theorem states that $\Psi_{N+1} \Psi_{N}^{-1}$ is a polynomial of degree 1 , and hence, for some constant matrices $R_{N}^{0}$ and $R_{N}^{1}$ we must have

$$
\Psi_{N+1} \Psi_{N}^{-1}=R_{N}(x)=R_{N}^{0}+x R_{N}^{1} .
$$

From the fact that $\operatorname{det}\left(R_{N}\right)$ does not depend on $x$ (last property of Theorem 2.2), we know that $R_{N}^{-1}(x)$ is a polynomial of degree at most 1 as well (this is easy if one considers the expression of the inverse of a matrix using the comatrix).

Comparing the asymptotics of $\Psi_{N+1}$ and $R_{N}(x) \Psi_{N}$ term by term in the expansion in inverse powers of $x$ and after some elementary algebra, one obtains (Ref. 20, Appendix A)

$$
R_{N}(x)=E_{\alpha_{0}} x+R_{N, 0} \quad \text { and } \quad R_{N}^{-1}(x)=E_{1} x+R_{N, 0}^{-1} .
$$

Here, we have introduced the notation $\alpha_{0}=r_{N}+1$ which corresponds to the index of the column where the coefficient $x^{-1}$ is to be found in the asymptotic of $\Psi_{N+1} \Psi_{N}^{-1}$. The notation $E_{j}$ denotes the matrix with 1 in position $j, j$ and 0 elsewhere. These notations are the standard notation used originally by Jimbo and Miwa in a Schlesinger transformation. The matrix $\left(R_{N, 0}\right)_{\alpha, \beta}$ is given by

$$
\begin{array}{cccc} 
& \beta=\alpha_{0} & \beta=1 & \beta \neq \alpha_{0}, 1 \\
\alpha=\alpha_{0} & \frac{-\left(Y_{N, 2}\right)_{\alpha_{0}, 1}+\sum_{\gamma \neq \alpha_{0}}\left(Y_{N, 1}\right)_{\alpha_{0}, \gamma}\left(Y_{N, 1}\right)_{\gamma, 1}}{\left(Y_{N, 1}\right)_{\alpha_{0}, 1}} & -\left(Y_{N, 1}\right)_{\alpha_{0}, 1} & -\left(Y_{N, 1}\right)_{\alpha_{0}, \beta} \\
\alpha=1 & \frac{1}{\left(Y_{N, 1}\right)_{\alpha_{0}, 1}} & 0 & 0 \\
\alpha \neq \alpha_{0}, 1 & -\frac{\left(Y_{N, 1}\right)_{\alpha, 1}}{\left(Y_{N, 1}\right)_{\alpha_{0}, 1}} & 0 & \delta_{\alpha, \beta}
\end{array}
$$

and $\left(R_{N, 0}^{-1}\right)_{\alpha, \beta}$ is given by 


$$
\begin{array}{cccc} 
& \beta=\alpha_{0} & \beta=1 & \beta \neq \alpha_{0}, 1 \\
\alpha=\alpha_{0} & 0 & \left(Y_{N, 1}\right)_{\alpha_{0}, 1} & 0 \\
\alpha=1 & -\frac{1}{\left(Y_{N, 1}\right)_{\alpha_{0}, 1}} & -\frac{-\left(Y_{N, 2}\right)_{\alpha_{0}, 1}}{\left(Y_{N, 1}\right)_{\alpha_{0}, 1}}+\left(Y_{N, 1}\right)_{1,1} & -\frac{\left(Y_{N, 1}\right)_{\alpha_{0}, \beta}}{\left(Y_{N, 1}\right)_{\alpha_{0}, 1}} \\
\alpha \neq \alpha_{0}, 1 & 0 & \left(Y_{N, 1}\right)_{\alpha, 1} & \delta_{\alpha, \beta} .
\end{array}
$$

While the formulas above might seem complicated, we will use the two important observations (that can be obtained easily with the definitions without the explicit formulas given above)

$$
\begin{gathered}
E_{\alpha_{0}} R_{N, 0}^{-1}+R_{N, 0} E_{1}=R_{N, 0}^{-1} E_{\alpha_{0}}+E_{1} R_{N, 0}=0, \\
R_{N}^{-1}(x) R_{N}^{\prime}(x)=R_{N, 0}^{-1} E_{\alpha_{0}} \text { does not depend on } x .
\end{gathered}
$$

The recurrence relation satisfied by the sequence $\left\{\tau_{N}\right\}$ is derived in the next theorem.

Theorem 3.2: Up to multiplication by functions that do not depend on the isomonodromic parameters (i.e., independent of the potentials $V_{1}$ and $V_{2}$ ), the following identity holds:

$$
\frac{\tau_{N+1}}{\tau_{N}}=\left(Y_{1}\right)_{1, \alpha_{0}} .
$$

Proof: The proof follows Ref. 21, but we report it here for convenience of the reader. Consider the following identity:

$$
\Psi_{N+1}=Y_{N+1} \Psi_{0}=R_{N} Y_{N} \Psi_{0}
$$

This implies that

$$
Y_{N+1}=R_{N} Y_{N}
$$

Taking the derivative with respect to $x$ gives

$$
Y_{N+1}^{-1} Y_{N+1}^{\prime}=Y_{N}^{-1} R_{N}^{-1} R_{N}^{\prime} Y_{N}+Y_{N}^{-1} Y_{N}^{\prime}
$$

Therefore, we have

$$
\begin{aligned}
d \log \tau_{N+1}-d \log \tau_{N} & =\operatorname{Res}_{x \rightarrow \infty} \operatorname{Tr}\left(\left(Y_{N}^{-1} R_{N}^{-1} R_{N}^{\prime} Y_{N}+Y_{N}^{-1} Y_{N}^{\prime}-Y_{N}^{-1} Y_{N}\right) d\left(\Psi_{0}\right) \Psi_{0}^{-1}\right) \\
& =\operatorname{Res}_{x \rightarrow \infty} \operatorname{Tr}\left(Y_{N}^{-1} R_{N}^{-1} R_{N}^{\prime} Y_{N} d\left(\Psi_{0}\right) \Psi_{0}^{-1}\right) .
\end{aligned}
$$

We now need to "transfer" the exterior derivative from $\Psi_{0}$ to $Y_{N}$. This can be done using that $\Psi_{N}=Y_{N} \Psi_{0}$, so that

$$
d \Psi_{N}=d\left(Y_{N}\right) \Psi_{0}+Y_{N} d\left(\Psi_{0}\right)
$$

Equivalently,

$$
Y_{N} d \Psi_{0} \Psi_{0}^{-1} Y_{N}^{-1}=d\left(\Psi_{N}\right) \Psi_{N}^{-1}-d Y_{N} Y_{N}^{-1} .
$$

Inserting these identities in the $\tau$ quotient, we obtain the relation

$$
d \log \tau_{N+1}-d \log \tau_{N}=\operatorname{Res}_{x \rightarrow \infty} \operatorname{Tr}\left(R_{N}^{-1} R_{N}^{\prime} d\left(\Psi_{N}\right) \Psi_{N}^{-1}-R_{N}^{-1} R_{N}^{\prime} d Y_{N} Y_{N}^{-1}\right) .
$$

The first term is residueless at $\infty$ since $d \Psi_{N} \Psi_{N}^{-1}$ is polynomial in $x$ and $R_{N}^{-1} R_{N}^{\prime}$ does not depend on $x$. Therefore, we are left only with 


$$
d \log \tau_{N+1}-d \log \tau_{N}=-\operatorname{Res}_{x \rightarrow \infty} \operatorname{Tr}\left(R_{N}^{-1} R_{N}^{\prime} d Y_{N} Y_{N}^{-1}\right)
$$

A direct matrix computation using the explicit form of $R_{N}$ yields

$$
d \log \tau_{N+1}-d \log \tau_{N}=d \log \left(\left(Y_{N, 1}\right)_{1, \alpha_{0}}\right)
$$

and hence

$$
\frac{\tau_{N+1}}{\tau_{N}}=\left(Y_{1}\right)_{1, \alpha_{0}} .
$$

The last equality is to be understood up to a multiplicative constant not depending on the parameters $u_{K}$ and $v_{J}$ in $\tau$.

In order to complete the first step, we need to express the entry $\left(Y_{1}\right)_{1, \alpha_{0}}$ in terms of the ratio of two consecutive partition functions. This is accomplished in the following section.

Theorem 3.3; For the matrix $\Gamma_{N}$ the asymptotic expansion at infinity (2.11) is such that

$$
\left(Y_{N, 1}\right)_{1, \alpha_{0}}=\left(v_{d_{2}+1}\right)^{S_{N}} h_{N}=\left(v_{d_{2}+1}\right)^{S_{N}} \frac{\mathcal{Z}_{N+1}}{\mathcal{Z}_{N}},
$$

where $S_{N}$ and $\alpha_{0} \in\left\{0,1, \ldots, d_{2}-1\right\}$ are defined by the following relation:

$$
N=d_{2} S_{N}+\alpha_{0}-1
$$

Proof: In order to compute $\left(Y_{N, 1}\right)_{1, \alpha_{0}}$, it is sufficient to compute the leading term of the expansion at $\infty$ appearing in the first row of the matrix $\Gamma_{N}$. Recalling the expression (2.13), we start by the following direct computation using integration by parts:

$$
\begin{aligned}
\iint_{\kappa} d z d w \pi_{N}(z) z^{i} w^{k-1} e^{-V_{1}(z)-V_{2}(z)+z w} & =\iint_{\kappa} d z d w \pi_{N}(z) e^{-V_{1}(z)} w^{k-1} e^{-V_{2}(w)} \frac{d^{i}}{d w^{i}}\left(e^{z w}\right) \\
& =(-1)^{i} \iint_{\kappa} d z d w \pi_{N}(z) e^{-V_{1}(z)+z w} \frac{d^{i}}{d w^{i}}\left(w^{k-1} e^{-V_{2}(w)}\right) \\
& =\iint_{\kappa} d z d w \pi_{N}(z) q_{d_{2} i+k-1}(w) e^{-V_{1}(z)-V_{2}(z)+z w}
\end{aligned}
$$

where $q_{d_{2} i+k-1}(w)$ is a polynomial of the indicated degree whose leading coefficient is $v_{d_{2}+1}^{i}$. The last right hand side is 0 if $d_{2} i+k-1<N$ because of orthogonality. If $d_{2} i+k-1=N$, the integral gives $v_{d_{2}+1}^{i} h_{N}$ by the normality conditions concerning our biorthogonal set. This computation allows us to expand the Cauchy transform of $\left(\Gamma_{N}\right)_{1, \alpha_{0}}$ near $\infty$ as follows:

$$
\begin{aligned}
\mathcal{C}\left(p_{N} w_{\alpha_{0}}(x)\right)= & \frac{1}{2 \pi i} \iint_{\kappa} d z d w \frac{\pi_{N}(z)}{z-w} w^{\alpha_{0}-1} e^{-V_{1}(z)-V_{2}(z)+z w} \\
= & -\sum_{i=0}^{S_{N}-1} \frac{1}{2 \pi i} \iint_{\kappa} d z d w \pi_{N}(z) \frac{z^{i}}{x^{i+1}} w^{\alpha_{0}-1} e^{-V_{1}(z)-V_{2}(z)+z w} \\
& +\frac{1}{2 \pi i} \frac{1}{x^{S_{N}+1}} \iint_{\kappa} d z d w \frac{\pi_{N}(z)}{x-z} z^{S_{N}} w^{\alpha_{0}-1} e^{-V_{1}(z)-V_{2}(z)+z w}+\mathcal{O}\left(x^{-S_{N}-2}\right) .
\end{aligned}
$$

By orthogonality the first sum vanishes term by term and the leading coefficient of the second term is $v_{d_{2}+1}^{S_{N}} h_{N}$.

We are finally in the position of formulating and proving the main theorem of the paper.

Theorem 3.4: The isomonodromic $\tau$ function and the partition function are related by 


$$
\forall N \in \mathbb{N}: \mathcal{Z}_{N}=\left(v_{d_{2}+1}\right)^{\Sigma_{j=0}^{N-1} S_{j}} \tau_{N}
$$

where we recall that $S_{j}$ is given by the decomposition of $j+1$ in the Euclidian division by $d_{2}$ : $S_{j}=E\left[(j+1) / d_{2}\right]$. A short computation of the power in $v_{d_{2}+1}$ gives

$$
\forall N \in \mathbb{N}: \mathcal{Z}_{N}=\left(v_{d_{2}+1}\right)^{d_{2}\left(\alpha_{N}\left(\alpha_{N}-1\right) / 2\right)+\alpha_{N}\left(N-\alpha_{N} d_{2}\right)} \tau_{N},
$$

where $\alpha_{N}=E\left[N / d_{2}\right]$.

Proof: Recall that the $\tau$ function is only defined up to a multiplicative constant not depending on $N$ nor on the coefficients $u_{k}$ and $v_{j}$. With this in mind and combining Theorems 3.2 and 3.3, we find that

$$
\frac{\tau_{N+1}}{\tau_{N}} \stackrel{\text { Theorem }}{=3.2}\left(Y_{1}\right)_{1, \alpha_{0}} \stackrel{\text { Theorem }}{=}{ }^{3.3}\left(v_{d_{2}+1}\right)^{S_{N}} \frac{\mathcal{Z}_{N+1}}{\mathcal{Z}_{N}},
$$

where $N=d_{2} S_{N}+\alpha_{0}-1$. Hence, for every $n_{0}$ :

$$
\tau_{N} \mathcal{Z}_{n_{0}}=\mathcal{Z}_{N} \tau_{n_{0}}\left(v_{d_{2}+1}\right)^{\sum_{j=n_{0}}^{N-1} S_{j}}
$$

One would like to take $n_{0}=0$ because it enables explicit computations. As we will prove now, there is a way of extending naturally all the reasoning down to 0 . Indeed, the RHP for $\Gamma_{N}$ (Theorem 2.1) is perfectly well defined for $N=0$ and has solution

$$
\Gamma_{0}=\left(\begin{array}{ccccc}
1 & \mathcal{C}_{0}(1) & \mathcal{C}_{1}(1) & \cdots & \mathcal{C}_{d_{2}-1}(1) \\
0 & 1 & 0 & \ldots & 0 \\
\vdots & \ddots & \ddots & \ddots & 0 \\
\vdots & \ddots & \ddots & \ddots & 0 \\
0 & 0 & \ldots & \ddots & 1
\end{array}\right) .
$$

Consequently, we can take

$$
\tau_{N} \mathcal{Z}_{0}=\left(v_{d_{2}+1}\right)^{\Sigma_{j=0}^{N-1} S_{j}} \mathcal{Z}_{N} \tau_{0}
$$

Also note that $\mathcal{Z}_{0} \equiv 1$ (by definition).

We can compute $\tau_{0}$ directly from Definition 3.2 because of the particularly simple and explicit expression of $\Psi_{0}=\Gamma_{0} \Psi_{0}$,

$$
d \ln \tau_{0}=\operatorname{res} \operatorname{Tr}\left(Y_{0}^{-1} Y_{0}^{\prime} d \Psi_{0} \Psi^{-1}\right) .
$$

We claim that this expression is identically zero (and, hence, we can define $\tau_{0} \equiv 1$ ); indeed,

$$
Y_{0}^{-1} Y_{0}^{\prime}=\left(\begin{array}{cccc}
0 & * & \cdots & * \\
0 & 0 & \cdots & 0 \\
\vdots & \vdots & \ddots & 0 \\
0 & 0 & \cdots & 0
\end{array}\right)
$$

and

$$
d \Psi_{0}(x) \Psi_{0}^{-1}(x)=\left(\begin{array}{cccc}
\star & 0 & \cdots & 0 \\
0 & \star & \cdots & \star \\
\vdots & \vdots & \ddots & \vdots \\
0 & \star & \cdots & \star
\end{array}\right)
$$

so that the trace of the product is always zero (even before taking the residue). 
The presence of the power in $v_{d_{2}+1}$ is due to a bad normalization of the partition function itself $\left(\mathcal{Z}_{N}\right)$ and can be easily canceled out by taking $v_{d_{2}+1}=1$ from the start (it is just a normalization of the weight function). This finding is consistent with the work of Bergere and Eynard ${ }^{2}$ since all their results concerning the partition function and its derivatives with respect to parameters have special cases for $u_{d_{1}+1}$ and $v_{d_{2}+1}$. It also signals the fact that the RHP is badly defined when $v_{d_{2}+1}=0$ because the contour integrals involved diverge and the whole setup breaks down. Indeed if $v_{d_{2}+1}=0$, this simply means that $V_{2}$ is a polynomial of lower degree, and thus the RHP that we should set up should be of smaller size from the outset.

\section{OUTLOOK}

In this article, we have restricted ourselves to contours going from infinity to infinity. This allows us to use integration by parts without picking up any boundary term. A natural extension of this work could be to see what happens when contours end in the complex plane and especially study what happens when the end points move (models with hard edges). This generalization is important in the computation of the gap probabilities of the Dyson model ${ }^{26}$ which correspond to a random matrix model with Gaussian potentials but with the integration restricted to intervals of the real axis.

\section{ACKNOWLEDGMENTS}

We would like to thank John Harnad for proposing the problem and Seung Yeop Lee and Alexei Borodin for fruitful discussions. This work was done at the University of Montréal at the Department of Mathematics and Statistics and the Centre de Recherche Mathématique (CRM) and O.M. would like to thank both for their hospitality. This work was partly supported by the Enigma European Network, Grant No. MRT-CT-2004-5652, by the ANR project Géométrie et intégrabilité en physique mathématique, Grant No. ANR-05-BLAN-0029-01, by the Enrage European Network, Grant No. MRTN-CT-2004-005616, by the European Science Foundation through the Misgam program, by the French and Japanese governments through PAI Sakurav, and by the Quebec government with the FQRNT.

\section{APPENDIX A: FACTORIZATION OF $\Psi_{N}$}

Starting from the definition of the last $d_{2}$ columns of $\Psi_{N}(2.19)$, we observe that

$$
\begin{aligned}
& \psi_{m}^{(k)}(x):=\frac{1}{2 i \pi} \int_{\check{\Gamma}_{k}} d s \iint_{\varkappa} \frac{\pi_{m}(z)}{x-z} \frac{V_{2}^{\prime}(s)-V_{2}^{\prime}(w)}{s-w} e^{-V_{1}(z)-V_{2}(w)+V_{2}(s)+z w-x s} d w d z \\
= & \sum_{p, q} v_{q+p} \frac{1}{2 i \pi} \iint_{\varkappa} \frac{\pi_{m}(z)}{x-z} w^{p-1} e^{-V_{1}(z)-V_{2}(w)+z w} \int_{\check{\Gamma}_{k}} d s s^{q-1} e^{V_{2}(s)-x s}=\sum_{p, q}\left(\hat{\Gamma}_{N}\right)_{m, p}\left(V_{0}\right)_{p, q}\left(W_{0}\right)_{q, k} \\
= & \left(\hat{\Gamma}_{N} V_{0} W_{0}\right)_{m, k} .
\end{aligned}
$$

This proves Theorem 2.2.

\section{APPENDIX B: BILINEAR CONCOMITANT AS INTERSECTION NUMBER}

We recall very briefly the result of Ref. 3 stating that 


$$
\left.\frac{V_{2}^{\prime}\left(\partial_{x}\right)-V_{2}^{\prime}\left(-\partial_{z}\right)}{\partial_{x}+\partial_{z}} w(x) f(z)\right|_{z=x}=\int_{\Gamma} \int_{\check{\Gamma}} \frac{V_{2}^{\prime}(\eta)-V_{2}^{\prime}(s)}{\eta-s} e^{x(\eta-s)-V_{2}(\eta)+V_{2}(s)}=2 i \pi \Gamma \# \check{\Gamma}=\text { const. }
$$

The last identity is obtained by integration by parts and shows that the bilinear concomitant is just the intersection number of the (homology classes) of the contours $\Gamma$ and $\check{\Gamma}$. More precisely, we get that

$$
\begin{aligned}
\frac{d}{d x} \int_{\Gamma} \int_{\Gamma} d s d \eta \frac{V_{2}^{\prime}(\eta)-V_{2}^{\prime}(s)}{\eta-s} e^{x(\eta-s)-V_{2}(\eta)+V_{2}(s)}= & \int_{\Gamma} \int_{\Gamma} d s d \eta\left(V_{2}^{\prime}(\eta)-V_{2}^{\prime}(s)\right) e^{x(\eta-s)-V_{2}(\eta)+V_{2}(s)} \\
= & \int_{\Gamma} \int_{\Gamma} d s d \eta \frac{\partial}{\partial \eta}\left(-e^{-V_{2}(\eta)}\right) e^{x \eta} e^{-x s+V_{2}(s)} \\
& -\int_{\Gamma} \int_{\Gamma} d \eta d s \frac{\partial}{\partial s}\left(e^{V_{2}(s)}\right) e^{-x s} e^{x \eta-V_{2}(\eta)} \\
= & x \int_{\Gamma} \int_{\Gamma} d s d \eta e^{x \eta-x s-V_{2}(\eta)+V_{2}(s)} \\
& -x \int_{\Gamma} \int_{\Gamma} d s d \eta e^{x \eta-x s-V_{2}(\eta)+V_{2}(s)}=0 .
\end{aligned}
$$

The matrix expression shows that the pairing is indeed a duality since the determinant is nonzero. The undressing matrix $\Psi_{0}$ (that was originally introduced in Theorem 2.3) is thus

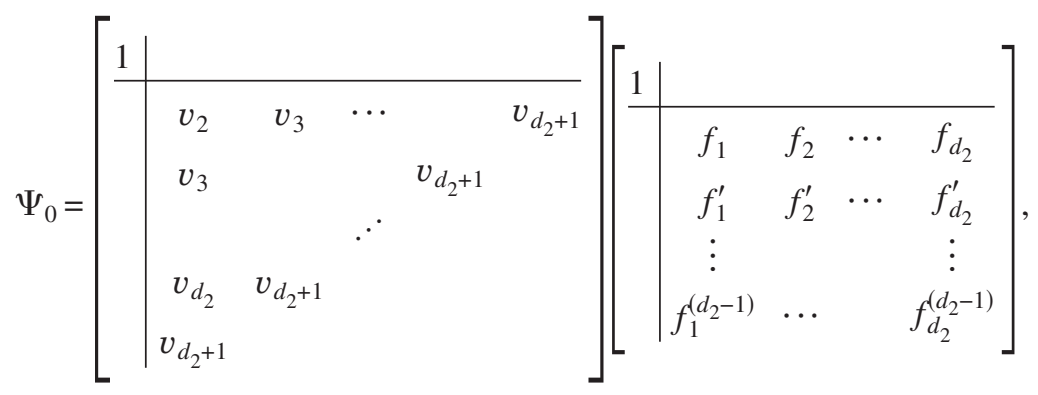

where the Wronskian sub-block in the second term is constructed by choosing $d_{2}$ homologically independent contour classes for the integrations $\check{\Gamma}$,

$$
f_{k}(x):=\int_{\check{\Gamma}_{k}} e^{-x s+V_{2}(s)} d s, \quad k=1, \ldots, d_{2} .
$$

The dressing matrix $\Psi_{0}$ exhibits a Stokes' phenomenon (of Airy's type) which is the inevitable drawback of removing the $x$ dependence from the jump matrix. We can now compute the jumps and see that it does not depend on $x$. For the $k$ th column we have

$$
\psi_{m}^{(k)}(x):=\frac{1}{2 \pi i} \int_{\check{\Gamma}_{k}} d s \int_{\varkappa} d z d w \frac{\pi_{m}(z) e^{-V_{1}(z)}}{x-z} \frac{V_{2}^{\prime}(s)-V_{2}^{\prime}(w)}{s-w} e^{-V_{2}(w)+V_{2}(s)+z w-x s}, \quad 1 \leq k \leq d_{2},
$$

giving

$$
\psi_{m}^{(k)}(x)_{+}=\psi_{m}^{(k)}(x)_{-}+\psi_{m}^{(0)}(x) \iint d s d w \frac{V_{2}^{\prime}(s)-V_{2}^{\prime}(w)}{s-w} e^{-V_{2}(w)+V_{2}(s)+x(w-s)}
$$




$$
=\psi_{m}^{(k)}(x)_{-}+\psi_{m}^{(0)}(x) \sum_{j=1}^{d_{2}} \varkappa_{\ell j}\left(\Gamma_{j}^{(y)} \# \check{\Gamma}_{k}\right),
$$

${ }^{1}$ Adler, M. and Van Moerbeke, P., "The spectrum of coupled random matrices," Ann. Math. 149, 921 (1999).

${ }^{2}$ Bergere, M. and Eynard, B., "Mixed correlation function and spectral curve for the 2-matrix model," J. Phys. A 39, 15091 (2006).

${ }^{3}$ Bertola, M., "Biorthogonal polynomials for two-matrix models with semiclassical potentials," J. Approx. Theory 144, 162 (2007).

${ }^{4}$ Bertola, M., Eynard, B., and Harnad, J., "Duality, biorthogonal polynomials and multi-matrix models," Commun. Math. Phys. 229, 73 (2002).

${ }^{5}$ Bertola, M., Eynard, B., and Harnad, J., "Differential systems for biorthogonal polynomials appearing in 2-matrix models and the associated Riemann-Hilbert problem," Commun. Math. Phys. 243, 193 (2003).

${ }^{6}$ Bertola, M., "Moment determinants as isomonodromic tau functions," Nonlinearity 22, 29 (2009); e-print arXiv:0805.0446.

${ }^{7}$ Bertola, M., Eynard, B., and Harnad, J., "Duality of spectral curves arising in two-matrix models," Theor. Math. Phys. 134, 27 (2003).

${ }^{8}$ Bertola, M. and Eynard, B., "The PDEs of biorthogonal polynomials arising in the two-matrix model," Math. Phys., Anal. Geom. 9, 23 (2006).

${ }^{9}$ Bertola, M., Eynard, B., and Harnad, J., "Partition functions for matrix models and isomonodromic tau functions," J. Phys. A 36, 3067 (2003).

${ }^{10}$ Bertola, M. and Gekhtman, M., "Biorthogonal Laurent polynomials, Töplitz determinants, minimal Toda orbits and isomonodromic tau functions," Constructive Approx. 26, 383 (2007).

${ }^{11}$ Bertola, M. and Mo, M. Y., "Isomodromic deformation of resonant rational connections," Int. Math. Res. Pap. 11, 565 (2005).

${ }^{12}$ Bertola, M., Eynard, B., and Harnad, J., "Semiclassical orthogonal polynomials, matrix models and isomonodromic tau functions," Commun. Math. Phys. 263, 401 (2006).

${ }^{13}$ Bertola, M., Harnad, J., and Its, A., "Dual Riemann-Hilbert approach to biorthogonal polynomials" (unpublished).

${ }^{14}$ Daul, J. M., Kazakov, V., and Kostov, I. K., "Rational theories of 2D gravity from the two-matrix model," Nucl. Phys. B 409, 311 (1993).

${ }^{15}$ di Francesco, P., Ginsparg, P., and Zinn-Justin, J., “2D gravity and random matrices,” Phys. Rep. 254, 1 (1995).

${ }^{16}$ Ercolani, N. M. and McLaughlin, K. T.-R., "Asymptotics and integrable structures for biorthogonal polynomials associated to a random two-matrix model," Physica D 152-153, 232 (2001).

${ }^{17}$ Eynard, B. and Mehta, M. L., "Matrices coupled in a chain: Eigenvalue correlations," J. Phys. A 31, 4449 (1998).

${ }^{18}$ Fokas, A., Its, A., and Kitaev, A., "The isomonodromy approach to matrix models in 2D quantum gravity," Commun. Math. Phys. 147, 395 (1992).

${ }^{19}$ Its, A. R., Tracy, A., and Widom, H., ““'Random words, Toeplitz determinants and integrable systems. II. Advances in nonlinear mathematics and science," Physica D 152-153, 199 (2001).

${ }^{20}$ Jimbo, M., Miwa, T., and Ueno, K., "Monodromy preserving deformation of linear ordinary differential equations with rational coefficients," Physica D 2, 306 (1981).

${ }^{21}$ Jimbo, M., Miwa, T., and Ueno, K., "Monodromy preserving deformation of linear ordinary differential equations with rational coefficients. II.," Physica D 2, 407 (1981).

${ }^{22}$ Kapaev, A. A., "The Riemann-Hilbert problem for the bi-orthogonal polynomials," J. Phys. A 36, 4629 (2003).

${ }^{23}$ Kontsevich, M., "Intersection theory on the moduli space of curves and the matrix Airy function," Commun. Math. Phys. 147, 1 (1992).

${ }^{24}$ Kuijlaars, A. B. J. and McLaughlin, K. T.-R., "A Riemann-Hilbert problem for biorthogonal polynomials," J. Comput. Appl. Math. 178, 313 (2005).

${ }^{25}$ Mehta, M. L. and Shukla, P., "Two coupled matrices: Eigenvalue correlations and spacing functions,” J. Phys. A 27, 7793 (1994).

${ }^{26}$ Tracy, C. and Widom, H., "Differential equations for Dyson processes," Commun. Math. Phys. 252, 7 (2004). 